\title{
ONE MODIFICATION OF $K-\varepsilon$ TURBULENT MODEL OF TWO-PHASE FLOWS
}

\author{
HOANG DUC LIEN \\ Agricultural University-Hanoi, Faculty of Mechanization \& Electrification \\ I. S. ANTONOV \\ Technical University-Sofia, Bulgaria, Hydro-aerodynamics Dept \\ NGUYEN THANH NAM \\ Polytechnic University HCM City, Mechanical Engineering Dept

\begin{abstract}
A modification of $k-\varepsilon$ turbulent model is described with application to the numerical investigation of two-phase turbulent jets. In difference of existed two-parameter models, new three-parameter modification have been suggested. In this case an additional equation for transportation of turbulent energy of admixture $-k_{p}$ is used together with equations of transportation of carrier- phase turbulent energy $-k_{g}$ and its dissipation $-\varepsilon$. with a new examination of the between-phases interaction forces determining jet flows.
\end{abstract} \\ Additional dissipation terms in the above mentioned equations are defined in connection
}

\section{Symbols}

$U_{i(i=p, g, o, m)}$ - Axial components of velocity

$V_{i(i=p, g, o, m)}$ - Radial components of velocity

$W_{i(i=p, g, o, m)}$ - Tangential components of velocity

$U_{i(i=p, g, o, m)}^{\prime}$ - Axial pulsative components of velocity

$V_{i(i=p, g, o, m)}^{\prime}$ - Radial pulsative components of velocity

$W_{i(i=p, g, o, m)}^{\prime}$ - Tangential pulsative components of velocity

$\chi_{i(0, m)}$ - Concentrates

$x, r, \theta$ - Coordinates of cylindrical coordinates system.

$k_{i(i=p, g)}$ - Kinetic energy of the motion

$\varepsilon$ - Dissipative velocity of the motion

$p, g, o, m$ - Symbols of admixture; gas; in the initial section; max. values

$R e, S c$ - Reynold and Smidth numbers

$\nu_{i(i=p, g)}$ - Turbulent viscosity

$R_{i}$ - Richarson's number

$C_{R}$ - Coefficient of aerodynamic resistance

$\sigma_{i(i=k, \varepsilon)}$ - Empirical constant. 


\section{Introduction}

A numerical modeling of two-phase turbulent jets by using $k-\varepsilon$ turbulent model have been done $1^{\text {st }}$ time by S. E. Elghobashi and T. W. Abou-Arab in [1-3] and the method have been developed by L. B. Gavin, V. A. Naumov, V. V. Shor in [4-8]. In these works to close the system equations of motion have been used two equations - for transportation of carrier-phase turbulent energy $-k_{g}$ and its' dissipation $-\varepsilon$.

In our works at the beginning of 90 's $[9,10]$ have done modification of the model by introducing an additional equation for transportation of turbulent energy of $2^{\text {nd }}$ phase - admixture - $k_{p}$ with the purpose to explain "two-fluid" character of the flow in turbulent model. The main concept is that the energy, lessened in admixture phase (particle) is transported into carrier (usually gas) phase. The model has not included an additional equation of velocity of turbulent dissipation of admixture because it can be considered by additional component in the equation of velocity of turbulent dissipation of carrier phase, same as performed in [11].

In this work for closing the system equations of motion the authors demonstrated a perfected modification of $k-\varepsilon$ model, called $k_{g}-k_{p}-\varepsilon$ model (or three-parameter model) with formulation of $k_{p}$ equation. An additional dissipated components are included, taking in consideration components of between-phases interaction forces, which are characterized for a two-phase turbulent jet and the numerical application in investigation of swirling two-phase turbulent jet.

\section{2. $k_{g}-k_{p}-\varepsilon$ Model equations}

Equations for transportation of turbulent (fluctuated) kinetic energy of carrier (gas) phase and of admixture have been received by developing equations in [1, 3] and considering some modification according to the theory of flow's boundary layer.

An equation for transportation of turbulent kinetic energy of carrier (gas) phase for swirling two-phase turbulent jet has a from as below:

$$
\begin{aligned}
U_{g} \frac{\partial k_{g}}{\partial x}+V_{g} \frac{\partial k_{g}}{\partial r}= & \frac{\partial}{r \partial r}\left[\frac{r \nu_{t g}}{\sigma_{k}}\left(\frac{\partial k_{g}}{\partial r}\right)\right]+\nu_{t g}\left[\frac{\partial U_{g}}{\partial r}\right]^{2} \\
& +\nu_{t g}\left[\frac{r \partial}{\partial r}\left(\frac{W_{g}}{r}\right)\right]^{2}+C_{R} k_{g}^{3 / 2} R_{i}-\varepsilon-\varepsilon_{p} .
\end{aligned}
$$

The above said equation is represented for stationary axis-symmetrical twophase flowing jet with unchanged density \& temperature of carrier phase $\left(T_{g}=\right.$ const \& $\rho_{g}=$ const). In difference with an usual from for single-phase flowing jets $k-\varepsilon$ model, this equation included an additional dissipated component $\varepsilon_{p}$, which 
describes two-phase character of the flow - dissipation of turbulent energy under influence of carried phase (admixture).

With the similar procedure for receiving equation for transportation turbulent energy (fluctuation) of carrier-phase, the authors come to the equation for transportation turbulent energy of $2^{\text {nd }}$ phase - admixture [9], taking into account some modifications: the first is: exclusion matrix of internal tensions - admitting that a dissipation of frictional tension is missed (this error can be compensated by frictional components in equation (2.1)). The second is concerning to an additional dissipated component $\varepsilon_{p}^{*}$, this component has a plus $\operatorname{sign}(+)$ because $\varepsilon_{p}^{*}$ led to generating turbulent energy of admixture from the above of carrier-phase. So, the equation for transportation turbulent energy of admixtures in swirling two-phase turbulent jet is:

$$
\begin{aligned}
U_{p} \frac{\partial k_{p}}{\partial x}+V_{p} \frac{\partial k_{p}}{\partial r}= & \frac{\partial}{r \partial r}\left[\frac{r \nu_{t p}}{\sigma_{k}}\left(\frac{\partial k_{p}}{\partial r}\right)\right]+\nu_{t p}\left[\frac{\partial U_{p}}{\partial r}\right]^{2} \\
& +\nu_{t p} \frac{r \partial}{\partial r}\left[\left(\frac{W_{p}}{r}\right)\right]^{2}+C_{R} k_{p}^{3 / 2} R_{i}+\varepsilon_{p}^{*}
\end{aligned}
$$

The components of the right side of equation (2.2) are: the $1^{\text {st }}$ is described for diffusive transportation of pulsated energy; the $2^{\text {nd }}$ determines turbulent energy's generating; the third is an additional dissipated component, through it can be considered influence of carrier-phase (gas) on the turbulent energy of admixture.

The same as in "two-parameter" model of turbulence, applied for two-phase flows [1-8], we used an equation for velocity of dissipation of turbulent pulsative energy. The from of that equation for swirling isothermal stationary axis-symmetrical two-phase flowing jets is as below:

$$
\begin{aligned}
U_{g} \frac{\partial \varepsilon}{\partial x}+V_{g} \frac{\partial \varepsilon}{\partial r}= & \frac{\partial}{r \partial r}\left[\frac{r \nu_{t}}{\sigma_{\varepsilon}}\left(\frac{\partial \varepsilon}{\partial r}\right)\right]-\Phi_{p}+C_{\varepsilon_{1}} \nu_{t g} \frac{\varepsilon}{k_{g}}\left[\frac{\partial U_{g}}{\partial r}\right]^{2} \\
& +C_{\varepsilon_{1}} \frac{\varepsilon}{k_{g}}\left[\frac{r \partial}{\partial r}\left(\frac{W}{r}\right)\right]^{2}-\frac{\varepsilon^{2}}{k_{g}}\left(C_{\varepsilon_{2}}-C_{\varepsilon_{3}} \chi_{m}\right)
\end{aligned}
$$

The $2^{\text {nd }}$ on the right side of the equation (2.3) is an additional dissipated component $\Phi_{p}$ which described influence of between-phase interaction forces on the equation of turbulent energy's dissipation.

According to Kolmogorov [1], between turbulent friction $\nu_{t}$ and turbulent (pulsative) kinetic energy exists the relation:

$$
\nu_{t}=C_{v} k^{0.5} L
$$

Applied for two-phase turbulent flows, taking into consideration of the two equations for turbulent energy of carrier-phase $k_{g}$ and of admixture $k_{p}$ the equations for turbulent friction of two phases can be written as follows:

$$
\nu_{t g}=C_{v} k_{g}^{0.5} L ; \quad \nu_{t p}=C_{v} k_{p}^{0.5} L
$$


A dissipation, occurred in the result of friction influences has the from:

$$
\varepsilon=C_{D} k_{g}^{0.5} L
$$

In the equations $(2.4),(2.5) C_{v}$ and $C_{D}$ are empirical constants, $L$ is a turbulent micro-ratio, proportional to radial dimension - of the flow, for example the relation between $L$ and difference of radial coordinates, where the carrier-phase velocities are 0.9 and 0.1 of its' max. values respectively in the same profile is:

$$
L=C_{\lambda}\left(r_{0.1}-r_{0.9}\right) \text {. }
$$

The empirical constants in the model are: $C_{v}=0.09 ; C_{D}=1 ; C_{\lambda}=0.625$ and others are given in the table 1 :

Table 1

\begin{tabular}{lllllll}
\hline$C_{v}$ & $C_{\varepsilon_{1}}$ & $C_{\varepsilon_{2}}$ & $C_{\varepsilon_{3}}$ & $\sigma_{k}$ & $\sigma_{\varepsilon}$ \\
\hline 0.009 & $\frac{1.44}{1.92}$ & $\frac{0.8}{1.9}$ & $\frac{1.0}{1.3}$ & $\frac{1.3}{2}$ \\
\hline
\end{tabular}

An additional dissipated component $\varepsilon_{p}$ is determined by the method described in $[8]$ as follows:

$$
\varepsilon_{p}=\frac{1}{\rho_{g}} \sum \overline{F_{i}^{\prime} V_{g i}^{\prime}}
$$

This component $\varepsilon_{p}$ considers an interaction of admixture on turbulent energy of carrier-phase, while taking into account and pulsation of between-phase interaction forces $f_{i}$, which are: resistive force $f_{A}$; Magnus's force $f_{M}$, Saffman's force $f_{s}$ for two-phase turbulent flows and in addition the forces of rotation $f_{w}$ and $f_{p w}$ for swirling two-phase turbulent jet. So, the additional dissipated component will be as below:

$$
\begin{aligned}
& \varepsilon_{p}=\varepsilon_{p A}+\varepsilon_{p M}+\varepsilon_{p S} \text { for Two-phase Turbulent Flows and } \\
& \varepsilon_{p}=\varepsilon_{p A}+\varepsilon_{p M}+\varepsilon_{p S}+\varepsilon_{p W}+\varepsilon_{p p W} \text { for Swirling Two-phase Turbulent Flows }
\end{aligned}
$$

where, an additional dissipated component $\varepsilon_{p A}$ from resistive force $f_{A}$ has a form:

$$
\varepsilon_{p A}=\frac{1}{\rho_{g}} \sum \overline{F_{A i}^{\prime} V_{g i}^{\prime}}=\frac{2 \rho_{p} \beta k_{g}}{\rho_{g}}\left[1-\exp \left(\frac{-B}{\beta \tau_{t}}\right)\right]
$$

$\tau_{t}=\left(B_{g} / \varepsilon\right)^{0.5}$ - timing micro-ratio, $B$ - empirical constant $(B=0.3), k_{g}=0.5 V_{g}^{2}$. An additional dissipated component from Magnus's force is determined from the equation:

$$
\varepsilon_{p M}=\frac{1}{\rho_{g}} \sum \overline{F_{M i}^{\prime} V_{g i}^{\prime}}=\frac{2 \rho_{p} k_{g} \lambda_{w}}{\rho_{g}}\left[1-\exp \left(\frac{-B}{\beta \tau_{t}}\right)\right]\left[\frac{W_{p}}{r}+0.5 \frac{\partial U_{g}}{\partial r}\right]
$$


An additional dissipated component from Saffman's force after re-working out becomes:

$$
\varepsilon_{p S}=\frac{1}{\rho_{g}} \sum \overline{F_{S i}^{\prime} V_{g i}^{\prime}}=2 k_{g} K_{s 1}\left[1-\exp \left(\frac{-B}{\beta \tau_{t}}\right)\right] \sqrt{\frac{\partial U_{g}}{\partial \tau}}
$$

For swirling two-phase turbulent jet it is necessary to add an additional dissipated components $\varepsilon_{p W}$ and $\varepsilon_{p p W}$, which are respectively [9]:

$$
\begin{aligned}
\varepsilon_{p W} & =\frac{1}{\rho_{g}} \sum \overline{F_{W i}^{\prime} V_{g i}^{\prime}}=\frac{2 \sqrt{2} \rho_{p} k_{W} k_{p} \sqrt{k_{g}}}{3 \rho_{g}} \\
\varepsilon_{p W W} & =\frac{1}{\rho_{g}} \sum \overline{F_{W i}^{\prime} V_{g i}^{\prime}}=\frac{2 \sqrt{2} k_{p W} k_{g}^{1.5}}{3} \\
k_{p} & =0.5 \sum \overline{V_{p i}^{\prime 2}} ; \quad k_{g}=0.5 \sum \overline{V_{g i}^{\prime 2}} .
\end{aligned}
$$

Same as for the carrier-phase, the additional dissipated components in equations for turbulent energy of admixture (particles) will be as flows [9]:

$$
\begin{aligned}
& \varepsilon_{p A}^{*}=\frac{1}{\rho_{g}} \sum \overline{F_{A i}^{\prime} V_{p i}^{\prime}}=2 \beta k_{g}\left[\exp \left(\frac{-B}{\beta \tau_{t}}\right)-\frac{k_{p}}{k_{g}}\right] \\
& \varepsilon_{p M}^{*}=\frac{1}{\rho_{g}} \sum \overline{F_{M i}^{\prime} V_{p i}^{\prime}}=\frac{2 \rho_{p} k_{g} \lambda_{w}}{\rho_{g}}\left[\exp \left(\frac{-B}{\beta \tau_{t}}\right)-\frac{k_{p}}{k_{g}}\right]\left[\frac{W_{p}}{r}+0.5 \frac{\partial U_{g}}{\partial r}\right] \\
& \varepsilon_{p W}^{*}=\frac{1}{\rho_{g}} \sum \overline{F_{W i}^{\prime} V_{p i}^{\prime}}=\frac{2 \sqrt{2} k_{p W} k_{p}^{1.5}}{3} .
\end{aligned}
$$

General from of additional dissipated components $\varepsilon_{p}^{*}$ in the equation for turbulent energy of admixture will have a from:

$$
\varepsilon_{p}^{*}=\varepsilon_{p A}^{*}+\varepsilon_{p M}^{*}+\varepsilon_{p W}^{*}
$$

An additional dissipated component $\Phi_{p}$ in the equation for velocity of turbulent energy's dissipation is determined by the function [8]

$$
\Phi_{p}=2 \frac{\nu}{\rho_{g}} \sum_{i} \sum_{k} \overline{\left(\frac{\partial F_{i}^{\prime}}{\partial x_{i}}\right)\left(\frac{\partial V_{g i}^{\prime}}{\partial x_{k}}\right)}
$$

The same as an additional dissipated component in the equation for turbulent energy $\varepsilon_{p}^{*}$, the values of $\Phi_{p}$ and its' components $\Phi_{p A}, \Phi_{p M}, \Phi_{p S}, \Phi_{p W}, \phi_{p p W}$ can be determined by following equation [8]: 


$$
\begin{aligned}
\Phi_{p} & =\Phi_{p A}+\Phi_{p M}+\Phi_{p S}+\Phi_{p W}+\Phi_{p p W} \\
\Phi_{p M} & =2 \beta \frac{\rho_{p}}{\rho_{g}} \frac{C_{t 1} \varepsilon}{C_{t 1}+\beta T_{E}}+\frac{2 \beta \nu C_{i 1}}{\rho_{g}\left(C_{t 1}+\beta T_{E}\right)} \sum_{j} \frac{\partial \rho_{p}}{\partial x_{j}} \frac{\partial k_{p}}{\partial x_{j}} \\
\Phi_{p A} & =2 \beta \frac{\rho_{p}}{\rho_{g}} \frac{C_{t 1} \varepsilon}{C_{t 1}+\beta T_{E}} \\
\Phi_{p S} & =2 K_{S 1} \sqrt{\frac{\partial U_{g}}{\partial r}} \frac{C_{t 1} \varepsilon}{C_{t 1}+\beta T_{E}} \\
\Phi_{p W} & =\frac{4 \sqrt{2}}{3} \frac{\rho_{p}}{\rho_{g}} \frac{K_{W} \sqrt{k_{p}} \beta \varepsilon T_{E}}{C_{t 1}+\beta T_{E}}
\end{aligned}
$$

where, the constants are defined as: $T_{E}=\lambda_{E} / U_{g}$ - integral-ratio on the time, $\lambda_{E}=C_{t 2} L$ - integral-ratio on distance, $C_{t 2}=0.575, C_{t 1}=1.48 \div 1.6$

\section{System equations \& numerical results of swirling two- phase turbulent jet}

The $k_{g}-k_{p}-\varepsilon$ model is used by authors for closing system equation, describing the motion of the swirling axis-symmetrical two-phase turbulent jet on the basis of two-fluid scheme, which in dimensionless form are as below:

$$
\begin{aligned}
& \frac{\partial\left(\bar{r} \bar{U}_{g}\right)}{\partial \bar{x}}+\frac{\partial\left(\bar{r} \bar{V}_{g}\right)}{\partial \bar{r}}=0 \\
& \frac{\partial\left(\bar{r} \bar{U}_{p}\right)}{\partial \bar{x}}+\frac{\partial\left(\bar{r} \bar{V}_{p}\right)}{\partial \bar{r}}=0 \\
& \frac{\partial \chi}{\partial \bar{x}}\left(\bar{r} \bar{U}_{p}\right)+\frac{\partial \chi}{\partial \bar{r}}\left(\bar{r} \bar{V}_{p}\right)=\frac{1}{\rho_{g}} \frac{\partial}{\partial \bar{r}}\left(\rho_{g} \bar{r} \frac{\bar{\nu}_{t p}}{S_{c}} \frac{\partial \chi}{\partial \bar{r}}\right) \\
& \frac{\partial \bar{U}_{g}}{\partial \bar{x}}\left(\bar{r} \bar{U}_{g}\right)+\frac{\partial \bar{U}_{g}}{\partial \bar{r}}\left(\bar{r} \bar{V}_{g}\right)+\frac{\partial \bar{P}}{\partial \bar{x}}=\frac{1}{\rho_{g}} \frac{\partial}{\partial \bar{r}}\left(\rho_{g} \bar{r} \frac{\bar{\nu}_{t g} \partial \bar{U}_{g}}{\partial \bar{r}}\right)-\bar{F}_{x} \bar{r} \\
& \frac{\partial \bar{U}_{P}}{\partial \bar{x}}\left(\bar{r} \bar{U}_{p}\right)+\frac{\partial \bar{U}_{p}}{\partial \bar{r}}\left(\bar{r} \bar{V}_{p} \frac{\bar{\nu}_{t p} \partial \chi}{S_{c} \partial \bar{r}}\right)=\frac{1}{\rho_{p}} \frac{\partial}{\partial \bar{r}}\left(\rho_{p} \bar{r} \frac{\bar{\nu}_{t p} \partial \bar{U}_{p}}{\partial \bar{r}}\right)+\bar{F}_{x} \bar{r} \\
& \frac{\partial \bar{W}_{g}}{\partial \bar{x}}\left(\bar{r}^{2} \bar{U}_{g}\right)+\frac{\partial \bar{W}_{g} \bar{r}}{\partial \bar{r}}\left(\bar{r} \bar{V}_{g}\right)=\frac{1}{\rho_{g}} \frac{\partial}{\partial \bar{r}}\left(\rho_{g} \bar{r}^{2} \bar{\nu}_{t g}\left(\frac{\partial \bar{W}_{g}}{\partial \bar{r}}-\frac{\bar{W}_{g}}{\bar{r}}\right)\right) \\
& \frac{\partial \bar{W}_{p}}{\partial \bar{x}}\left(\bar{r}^{2} \bar{U}_{p}\right)+\frac{\partial \bar{W}_{p} \bar{r}}{\partial \bar{r}}\left(\bar{r} \bar{V}_{p} \frac{\bar{\nu}_{t p}}{S_{c}} \frac{\partial \chi}{\partial \bar{r}} \bar{r}\right)= \\
& =\frac{1}{\rho_{p}} \frac{\partial}{\partial \bar{r}}\left(\rho_{p} \bar{r}^{2} \bar{\nu}_{t p}\left(\frac{\partial \bar{W}_{p}}{\partial \bar{r}}-\frac{\bar{W}_{p}}{\bar{r}}\right)\right)
\end{aligned}
$$




$$
\begin{aligned}
& \frac{\partial \bar{P}}{\partial \bar{x}}=\rho_{g} \frac{\bar{W}_{g}^{2}}{\bar{r}}+F_{y}, \\
& \frac{\partial \bar{k}_{g}}{\partial \bar{x}}\left(\bar{r} \bar{U}_{g}\right)+\frac{\partial \bar{k}_{g}}{\partial \bar{r}}\left(\bar{r} \bar{V}_{g}\right)=\frac{1}{\rho_{g}} \frac{\partial}{\partial \bar{r}}\left(\rho_{g} \bar{r} \frac{\bar{\nu}_{t g}}{\sigma_{k}} \frac{\partial \bar{k}_{g}}{\partial \bar{r}}\right)+ \\
& +\bar{\nu}_{t g}\left(\frac{\partial \bar{U}_{g}}{\partial \bar{r}^{\prime}}\right)+C_{R} \bar{k}_{g}^{3 / 2} R_{i}-\bar{\varepsilon}-\bar{\varepsilon}_{p}^{*} \\
& \frac{\partial \bar{k}_{p}}{\partial \bar{x}}\left(\bar{r} \bar{U}_{p}\right)+\frac{\partial \bar{k}_{p}}{\partial \bar{r}}\left(\bar{r} \bar{V}_{p} \frac{\bar{\nu}_{t p}}{S_{c}} \frac{\partial \bar{k}_{p}}{\partial \bar{r}} \bar{r}\right)=\frac{1}{\rho_{p}} \frac{\partial}{\partial \bar{r}}\left(\rho_{p} \bar{r} \frac{\bar{\nu}_{t p}}{\sigma_{k}} \frac{\partial \bar{k}_{p}}{\partial \bar{r}}\right)+ \\
& +\bar{\nu}_{t p}\left(\frac{\partial \bar{U}_{p}}{\partial r}\right)+C_{R} \bar{k}_{p}^{3 / 2} R_{i}+\bar{\varepsilon}_{p}^{*}, \\
& \frac{\partial \bar{\varepsilon}}{\partial \bar{x}}\left(\bar{r} \bar{U}_{g}\right)+\frac{\partial \bar{\varepsilon}}{\partial \bar{r}}\left(\bar{r} \bar{V}_{g}\right)=\frac{1}{\bar{\rho}_{g}} \frac{\partial}{\partial r}\left(\rho_{g} \bar{r} \frac{\bar{\nu}_{t g}}{\sigma_{\varepsilon}} \frac{\partial \bar{\varepsilon}}{\partial \bar{r}}\right)+C_{\varepsilon 1} \bar{\nu}_{t g} \frac{\bar{\varepsilon}}{\bar{k}_{g}}\left(\frac{\partial \bar{U}_{g}}{\partial \bar{r}}\right)^{2} \\
& +C_{\varepsilon_{1}} \frac{\bar{\varepsilon}_{\bar{k}}}{\bar{k}_{g}}\left[\bar{r} \frac{\partial}{\partial \bar{r}}\left(\frac{\bar{W}_{g}}{\bar{r}}\right)\right]^{2}-C_{\varepsilon 2} \frac{\bar{\varepsilon}^{2}}{\bar{k}_{g}}-\bar{\Phi}_{p}
\end{aligned}
$$

where: $\bar{x}=x / r_{0} ; \quad \bar{U}_{i}=U_{i} / U_{0} ; \quad \bar{V}_{i}=V_{i} / V_{0} ; \quad \bar{k}_{g}=k_{g} / U_{0}^{2} ; \quad \bar{k}_{p}=k_{p} / U_{0}^{2}$, $\bar{P}=P /\left(\rho_{g} U_{0}^{2}\right) ; \bar{W}_{i}=W_{i} / W_{0} ; \quad \bar{\nu}_{t i}=\nu_{t i} /\left(U_{0} r_{0}\right) ; \quad \bar{\varepsilon}=\varepsilon r_{0} / U_{0}^{3} ; \bar{\Phi}_{p}=\Phi_{p} r_{0}^{2} / U_{0}^{4} ;$ $\bar{F}=F r_{0} /\left(\rho_{g} U_{0}^{2}\right)$

Boundary Conditions:

- In the axis of the flow $(r=0)$.

$$
\frac{\partial \bar{U}_{g}}{\partial \bar{r}}=\frac{\partial \bar{U}_{p}}{\partial \bar{r}}=0, \quad \frac{\partial \chi}{\partial \bar{r}}=0 ; \quad \frac{\partial \bar{k}_{p}}{\partial r}=\frac{\partial \bar{k}_{g}}{\partial \bar{r}}=\frac{\partial \bar{\varepsilon}}{\partial \bar{r}}=0
$$

$\bar{W}_{p}=\bar{W}_{g}=0 ; \bar{V}_{p}=\bar{V}_{g}=0$

- On the boundary layer $\left(U_{i}=0\right)$

$\bar{W}_{g}=\bar{W}_{p}=0 ; \bar{U}_{p}=\bar{U}_{g}=0 ; \bar{k}_{p}=\bar{k}_{g}=0, \bar{\varepsilon}=\bar{P}=0$.

Initial Values:

$W_{0}=S_{0} r_{0}$

$U_{i 0}=U_{0} ; \quad \chi_{0}=\rho_{p} / \rho_{g} ; \quad k_{i 0}=\left(0.1 U_{i 0}\right)^{2} ; \quad V_{i 0}=0 ; \quad \varepsilon_{0}=C_{D} k_{g 0}^{1.5} / L ;$

where $S_{0}$ is initial swirling factor.

The system of 11 equations with 11 unknowns $\left(\bar{U}_{i}, \bar{V}_{i}, \bar{W}_{i}, \bar{k}_{i(i=p, g)} ; \bar{\varepsilon} ; \chi, \bar{P}\right)$ is numerically solved with finite difference method using Duifort-Frankel differential scheme [9].

On Fig. 1 showing the numerical results of $U_{g m}$ and $U_{p m}$, receiving by solving before said system equation for the case: $S_{0}=0, \chi_{0}=0.5 \& 1.0 ; D_{p}=50 \mu \mathrm{m}$; $U_{0}=19 \& 21 \mathrm{~m} / \mathrm{s}$, comparison with experiments of Shriber [8]. The prediction and experiments show good agreement, which illustrates the capability of the model in predicting complex flows. 


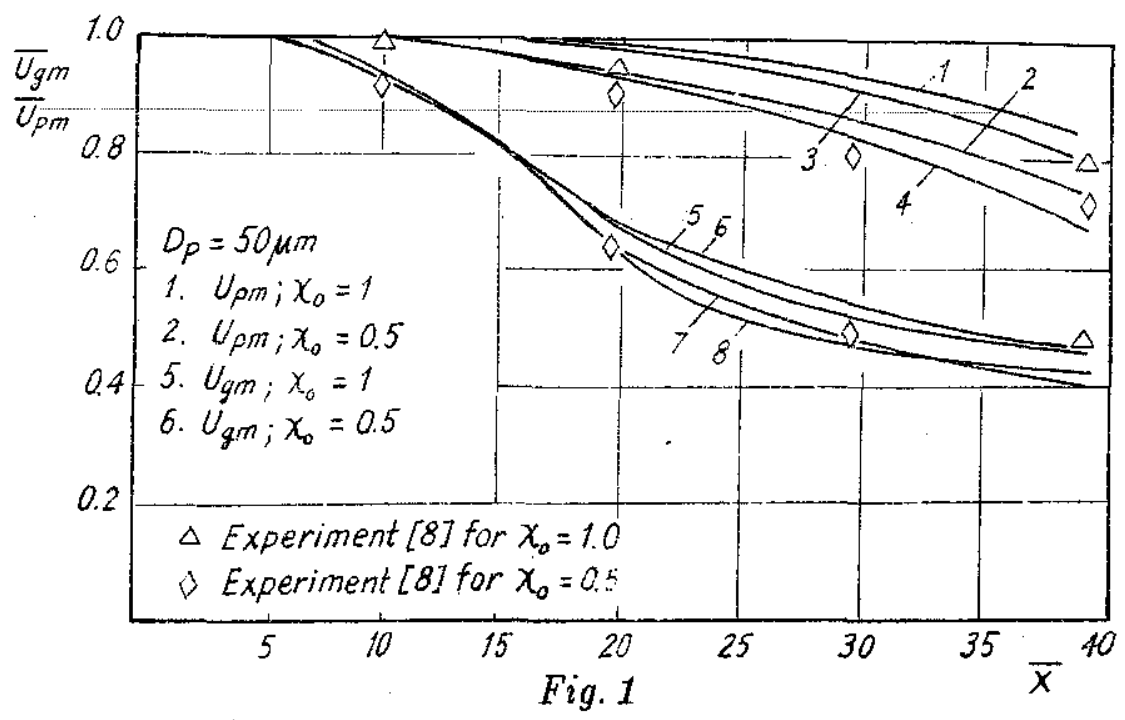

\section{Conclusion}

The $k_{g}-\dot{k}_{p}-\varepsilon$ model proposed in this paper shows that from physical point of view the $2^{\text {nd }}$ phase (admixture) in two-phase flows can be considered as independent phase with own characters and the relation between two phases is existed through between-phases interaction forces. The numerical computation can be used for predicting complex two-phase flows.

The work was supported by Vietnam National Foundation for Basis Research on Natural Science. These aids are gratefully acknowledged.

\section{References}

1. Elghobashi S. E. Particle-Laden turbulent flow direct simulation and closur models, Appl. Sci. Res. V.48, 3/4, 1991, p.p. $301-314$ (1992).

2. Elghobashi S. E., T. W. Abou-Arab. A second-order turbulence model for two-phase flow, Int. J. Heat Transfer, 5, 1982, p.p. 219-224.

3. Elghobashi S. E., T. W. Abou-Arab. A two-equation turbulence model for two-phase flow, Phys. Fluids, 26, 4, 1993, p.p. 931-938.

4. Гавин Л. Б., Наумов В. А. Турбулентная двухфазнфя струя и ее численное исследование, ИФЖ, 44, 6, 1983.

5. Гавин Л. Б., Наумов В. А., Щор В. В. Численое исследование газовой струи с тяжелыьи частицами на основе двухпараметрической модели турбулентности, ПМТФ 1, 1984. стр. 62-67.

6. Гавин Л. Б., Мульги А. С., Щор В. В. Численое и экспериментальное исследование неизотермической турбулентной струи с тяжелый примесью, ИФЖ, Т.5 1986г., стр. 735-743. 
7. Яценко В. П., Моронова М. В. Расчет движения мелкодисперсных частиц в турбулентном пограничном слое, в кн. Турбул. Двухфазно теч. и тех.

8. Гавин Л. Б., Щрайбер А. А. Турбулентные течения газа с частицами, Итоги науки и тех., серю Мех. Жидкости и газа (ВИНИТИ), 1991.

9. Antonov I. S., N.T. Nam. Numerical methods for modeling of a two-phase turbulence swirling jets, Intern. Symp. on Hydro-and Aerodinamics in Marine Engineering, HADMAR'91 Nov. 1991, Varna. Proc. vol.1, 34-1 -35-5.

10. Antonov I. S., N. T. Nam, H. D. Lien. Two-phase turbulent jet. $K-\varepsilon$ model, Proc. of the 4th Workshop of Applied Mechanics. 28th April 1994, Center of Computational Mech., HUT, Ho-Chi-Minh city Vietnam, p.p. X/1-X/7.

11. Лаундер Б. Е. Модели замыкания для напряжения третье поколение, в кн. Турбул. сдвиг. течения, Т.1 М., Машинностроение, 1982, стр. 270279.

Received October 20, 1997

\section{VỀ MộT SƯ BIẾN DANG CỬA MÔ HİNH $K-\varepsilon$ ĐỐI VỚI DÒNG CHẢY RỐI HAI PHA}

Sự biến dạng mô hình $K-\varepsilon$ của sự rối, được xem xét, ứng dụng trong việc nghiên cứu dòng phun rối hai pha. Sự khác nhau đối mô hình 2 thông số đang tồn tại là ở đây được đưa vào sự biến dạng với mô hình 3 thông số mới. Trong đó ngoài 2 phương trình về năng lượng mạch động của pha kéo theo $K_{g}$ và sự tán mạn của nó $\varepsilon$, được đưa vào phương trình bổ sung về năng lượng rối của pha tạp chất $-K_{p}$. Những thành phần tản mạn bồ sung ơ các phương trình trên được xác định trên cơ sở về một cách nhìn nhận mới đối với việc xác định các lực trong dòng phun của sự tương tác giữa các pha với nhau. 